\title{
Injection into Electron Plasma Traps
}

\author{
Vladimir Gorgadze, Thomas A. Pasquini, Joel Fajans, Jonathan S. Wurtele \\ Department of Physics, UC Berkeley, Berkeley, CA 94720
}

\begin{abstract}
.
Computational studies and experimental measurements of plasma injection into a MalmbergPenning trap reveal that the number of trapped particles can be an order of magnitude higher than predicted by a simple estimates based on a ballistic trapping model. Enhanced trapping is associated with a rich nonlinear dynamics generated by the space-charge forces of the evolving trapped electron density. A particle-in-cell simulation is used to identify the physical mechanisms that lead to the increase in trapped electrons. The simulations initially show strong two-stream interactions between the electrons emitted from the cathode and those reflected off the end plug of the trap. This is followed by virtual cathode oscillations near the injection region. As electrons are trapped, the initially hollow longitudinal phase-space is filled, and the transverse radial density profile evolves so that the plasma potential matches that of the cathode. Simple theoretical arguments are given that describe the different dynamical regimes. Good agreement is found between simulation and theory.
\end{abstract}

\section{INTRODUCTION}

Pure electron plasmas confined in Malmberg-Penning traps have been the subject of extensive theoretical and experimental investigations over the last two decades [1]. These traps have an axial magnetic field which confines electrons radially and an electrostatic well which confine them axially (see Fig. 1). Electrons are injected into the trap by lowering the one wall of the electrostatic well, thereby allowing electrons to flow from the cathode trap to the confinement region. An electron column subsequently forms between the negatively-biased cathode and the far wall of the electrostatic well. Next, the column is pinched off from the cathode by restoring the near wall of the electrostatic well. The electrons in confinement region are thus trapped, ending the injection phase of the experimental cycle. If the injection time is sufficiently long, it has been observed that the number of electrons increases, and their phase-space distribution evolves, until the electron column's electrostatic potential matches that of the cathode bias potential. After injection, experiments on a wide-range of phenomena, from vortex dynamics [2] to collisional relaxation [3] are conducted. The plasmas created in these traps are highly reproducible. Surprisingly, the injection process itself has not been carefully studied in previous experimental and theoretical investigations.

Our experiments and particle-in-cell (PIC) simulations show that the number of trapped electrons is much higher than expected from a straightforward picture of the injection process. In this straightforward picture, the trapping process is purely ballistic. Electrons leave the cathode, stream across the confinement region, bounce from the far electrostatic wall, and return to be reabsorbed by the cathode. The restoration of a negative bias to the confinement wall near the cathode isolates the cathode from the main 


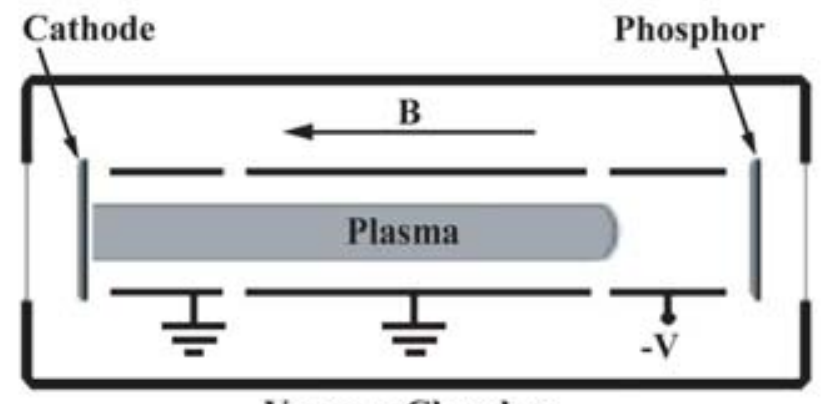

Vacuum Chamber

FIGURE 1. Malmberg-Penning traps consist of a series of collimated conducting cylinders, or gates, aligned along a strong magnetic field. Electron plasmas are confined in these traps by appropriately biasing the trap cylinders to form an axial electrostatic well. Radial confinement is provided by a strong magnetic field. Electrons are injected into the trap by grounding an 'inject' gate near the cathode, and allowing electrons from the negatively biased cathode to enter the trap as shown. The plasma is imaged by briefly grounding the 'dump' gate, accelerating the electrons toward the phosphor screen, and recording the image with a CCD camera.

plasma column. The electrons thereby confined are not in any sense trapped before the column is pinched; these are just the electrons that happen to be transiting the trapping region the instant the column is pinched. The density of these transiting electrons will be $\sim 2 J / q v$, where $q$ is the electron charge, and $v$ is the average speed of the electrons. We find that the actual number of trapped electrons is an order of magnitude higher than predicted by estimates based on this ballistic trapping. Some other mechanism must be trapping electrons in the confinement region, and these electrons must be genuinely trapped in the sense that they are confined to the central column region, rather than reabsorbed at the cathode, before the column is pinched. Thus, the physics of the injection process goes beyond a model consisting of laminar, time independent electron motion, for, in such a model, electrons could not be trapped. This paper elucidates the critical role of self-fields, with their rich nonlinear physics, in the process by which the trapped charge continues to increase over hundreds of ballistic transit times.

Several mechanisms exist that could explain the increase in density beyond the ballistic limit. One such mechanism is trapping through collision with other electrons or with background neutral gas. If an electron loses kinetic energy in a collision it will not be able to return to the cathode and will be on a trapped orbit. Calculated collision rates and experimental measurements indicate, however, that this mechanism is far too slow. Another mechanism could be a small inward displacement of the electrons. Most Malmberg-Penning traps use a spiral-wound tungsten filament that is increasingly negative in the center. Thus, an electron that moved inward might not have enough kinetic energy to return to the cathode, and would be trapped. However, most of our experiments were done with an unusual equipotential cathode that could not trap electrons by this mechanism.

Simulations of this system are straightforward, and suggest that multiple dynamic processes lead to the trapping of electrons during the injection. Very roughly, the processes tend to occur at different temporal stages of the trapping:

In the first stage, electrons accelerate into the initially empty trapping region, reflect 
off of the potential barrier and return to the cathode. No trapping occurs during this ballistic filling of the trap.

In the second stage, the stream of electrons leaving the cathode interacts with the counter-propagating electrons that have reflected off the end of the trap. A two-stream interaction develops which distorts the equilibrium fields in the trap. Localized timedependent perturbations in the electrostatic potential grow to the point where they extract energy from localized groups of electrons. These electrons are unable to return to the cathode and are trapped.

In the third stage, electrons which have lost energy interact with the transiting charges, thereby causing the development of a novel, soliton-like travelling cavitation, or bubble, so-named for the appearance of a large bubble in phase space. This bubble produces strongly inhomogeneous plasma self-fields, which traverse the length of the trap many times, decelerating and trapping electrons.

In the fourth stage, which overlaps significantly with the prior stage, the effective current in the trapping region exceeds a critical value and a virtual cathode develops near the physical cathode. The size and position of the virtual cathode are affected by the phase space dynamics. As the cavitation dies off, the oscillations of the virtual cathode continue trapping electrons, albeit at a slower rate than before. In this final stage, the plasma column develops a profile determined not by the current emission from the cathode, but by the matching condition between the plasma potential and the cathode potential. Consequently, a plasma created by a filament cathode (which has a parabolic potential) maintains an approximately constant density. A plasma produced by an equipotential cathode emitting uniformly will hollow significantly as its potential begins to match that of the cathode. The hollow column is then subject to the diocotron instability, which takes a somewhat different form when it occurs in contact with an emitting cathode. In the following sections we give an overview of the experiment and simulation and describe the trapping regimes.

\section{EXPERIMENT AND SIMULATION}

Experimental studies of trapping during injection have been performed on two Malmberg-Penning traps, as shown in Fig. 1 The bulk of the data was collected on the Berkeley Photocathode trap [4], in which the cathode is an illuminated photoemissive material held at a constant potential [5]. The experiments were repeated on a more typical trap where the cathode is a heated tungsten filament and has a parabolic potential profile, and similar results were found. The only significant difference observed occurs in the late time development of the plasma column; the column hollowing and subsequent instabilities on the photocathode machine are absent on the machine with the parabolic cathode. This discrepancy is understood to be a result of the potential matching condition between the plasma column and the cathode.

In the photocathode machine, a $1.0 \mathrm{~cm}$ diameter circular section of the cathode is illuminated with light of a constant intensity. For a typical injection time of $30 \mu \mathrm{s}$ with a $-5 \mathrm{~V}$ photocathode bias, the plasma column has a density of $1 \times 10^{7} \mathrm{~cm}^{-3}$. In the thermal emission machine, the filament emits over a $1.1 \mathrm{~cm}$ diameter. For a typical injection 
time of $1 \mathrm{~ms}$ with a $-10 \mathrm{~V}$ bias, the plasma column has a density of $1 \times 10^{7} \mathrm{~cm}^{-3}$. In both machines, the plasma temperature is approximately $1 \mathrm{eV}$, corresponding to a Debye length of $0.23 \mathrm{~cm}$.

Measurements of the electron population within the trapping region are made as follows. The electron column is injected for the desired time by grounding the trap segment closest to the cathode, the inject gate. The electron population is then isolated from the cathode by lowering the potential on the inject gate to a large negative value. This process traps approximately $80 \%$ of the electrons present in the trapping region. The plasma column is then allowed to escape by grounding the trap segment closest to the phosphor screen. Electrons are accelerated onto the phosphor, and the resulting light is recorded by a CCD camera. Analysis of the pattern gives the number of charges in the trapping region. This procedure cannot distinguish between electrons which were electrostatically trapped during the injection process and those which were circulating through the trapping region when the inject gate was lowered.

Simulations were performed using the PIC simulation XOOPIC [6]. Owing to computational expense, we limit our simulations to the short time evolution of the system $(<1 \mathrm{~ms})$. The electrostatic code is two-dimensional with an assumed $(r, z)$ dependence for the electrostatic field. The system is assumed to possess azimuthal symmetry. The gyroradius in the experiment is of order $10^{-3} \mathrm{~cm}$, and typical plasmas have a Debye length of $10^{-1} \mathrm{~cm}$. We thus make the further approximation that the gyromotion can be ignored. Electrons are therefore limited to motion in the axial direction, along the magnetic field lines. A uniform mesh is applied in both the axial and radial coordinates. The radial distribution still evolves, since the trapping dynamics depends on both self-fields and external fields, and, hence, on the radius.

Simulations have been made for both the equipotential cathode and the parabolic cathode. It is assumed in both cases that electron emission is constant over the area of the cathode. The experimental value of the current was used in all simulations. The temperature of the emitted electrons (assumed Maxwellian distribution) was taken as $T=0.1 \mathrm{eV}$ to fit the experimental data.

The experimental and simulation results are plotted in Fig. 2, where the lower curves correspond to cathode with flat potential and the upper curves are for parabolic cathode potential. The simulations reproduce the experimental results and, importantly, provide detailed information on the phase space density. The insights gained from simulations lead to an understanding of the nonlinear processes in the trapping.

\section{Ballistic Filling}

As previously noted, no electron trapping occurs in the first stage of the injection process. Electrons emitted from the cathode traverse the trapping region, reflect from the negatively biased end gate and return to the cathode to be reabsorbed. The number of electrons in the trapping region increases linearly with time until the first electrons to enter the trapping region are reabsorbed at the cathode. At this point, an equilibrium number of trapped charges is reached, depending only on the cathode current, the thermal distribution of emitted electrons, and the trap length. Calculations predict an elec- 


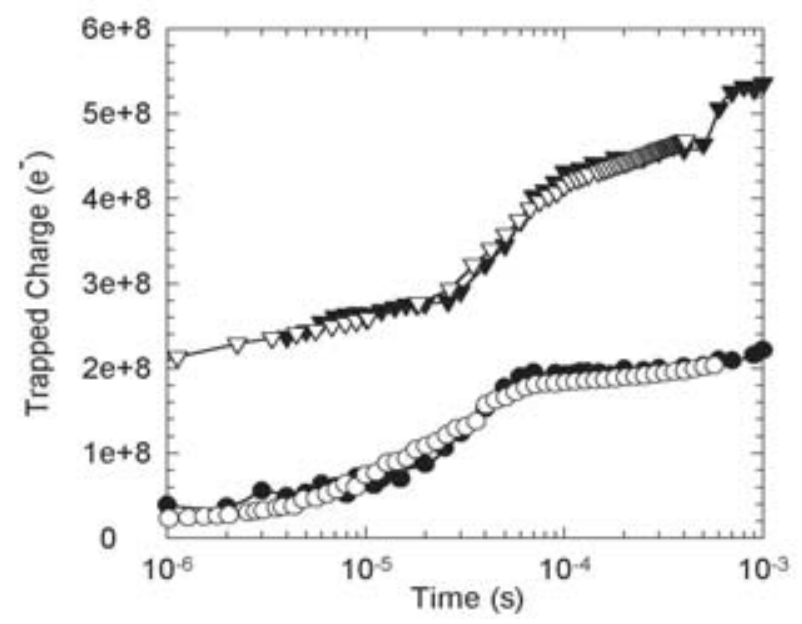

FIGURE 2. The total number of trapped electrons as a function of time for an equipotential cathode (circles) and a tungsten filament (triangles). Experimental data are shown in black, simulation data in white. Very short injection times $(<1 \mu \mathrm{s})$ are explained by a non-trapping model. Beyond $1 \mu \mathrm{s}$, however, instabilities in the injection beam lead to charge trapping.

tron population of $2 \times 10^{7}$ for the photocathode trap and of $2.1 \times 10^{7}$ for the parabolic cathode trap.

Simulation results shown in Fig. 3, corresponding to the first bounce period of $0.5 \mu \mathrm{s}$, confirm that the total charge in the trapping region increases linearly to the value predicted by ballistic trapping. Electrons then continue to cycle in and out of the trap region. In phase space, also shown in Fig. 3A - 3D, the electron orbits correspond to a split ring trajectory. The phase space plot shows that none of the charges in the trapping region are actually trapped.

The equilibrium number of electrons in the trap depends only on the cathode current, the thermal distribution of emitted electrons, and the trap length. The velocity of the particles remains almost constant throughout the trap (Fig. 3D) and can be found from conservation of energy (neglecting self-fields) to be $v_{e}=1.3 \times 10^{6} \mathrm{~m} / \mathrm{s}$. Thus, the transit time for a single particle is $\tau_{\text {bounce }}=2 \times L_{\text {trap }} / v_{e} \approx 3.8 \times 10^{-7} \mathrm{~s}$. Taking the injected current $J_{N}=5 \times 10^{13} \mathrm{e} / \mathrm{s}$, calculations and simulations are both within $10 \%$ experimental value of $2.4 \times 10^{7} e$ for the number of trapped electrons at $t=\sim 1 \mu \mathrm{s}$ (the earliest time for which data could be taken). This "equilibrium" value is, in fact, only maintained for a few bounce times. The dynamics quickly becomes more complicated, with the streams of electrons emitted from and returning to the cathode interacting with each other, as is next discussed. 


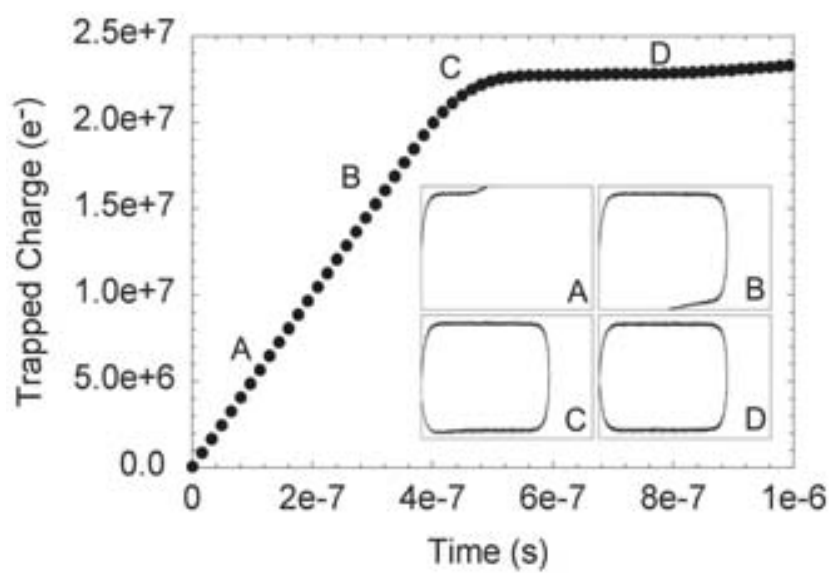

FIGURE 3. The total number of trapped electrons as a function of time during the first stage of injection with inset plots of the $z-v_{z}$ phase space. The labels A-D match the times of corresponding phase-space plots. During this first injection stage, no electrons are trapped.

\section{Two-Stream Trapping}

Directly after the initial ballistic trapping, the number of particles in the trap doubles, and an oscillation develops with a period of roughly one bounce time, within $\sim 10$ bounce times. This is shown in Fig. 4 along with the phase space distribution at three different times. The simulation shows that as phase space structure appears, the potential becomes time dependent, and particles are trapped. Energy loss is needed for trapping, since an electron that circuits that trap and returns with the same or more energy will be reabsorbed at the cathode. Collisional losses are, for the plasmas in question, too slow to account for the trapping, and thus collisionless mechanisms must be examined. An obvious candidate is the development of a two-stream interaction between the electrons leaving and returning to the cathode. Indeed, the simulations reproduce the experimental results and indicate that this is the mechanism for initial trapping.

Simulations confirm that a two-stream interaction develops shortly after the initial filling stage. From the phase space plots Fig. 4B,C, it is evident that some electrons lose energy as they circuit the trap. As these electrons are unable to be reabsorbed at the cathode, there results a net increase in the number of trapped electrons. The plasma evolves by continuing to trap fresh electrons and subjecting the previously trapped electrons to further energy loss. Thus, the originally hollow longitudinal phase space begins to fill-in. The dynamic builds on itself, as initially small time-dependent fields grow ever larger.

The importance of two-stream interactions in this stage is demonstrated by numerical studies. We can eliminate two stream interactions by performing a simulation in which twice the charge is emitted from the cathode, but all the electrons are absorbed at the reflecting end of the trap. In this way the charge density is the same as in the the reflecting case, but there is only one stream of electrons. In this case, the electron stream remains quiescent, showing that energy loss and trapping are connected to the existence of two counter propagating streams of electrons. Simulations also indicate growth of Langmuir 


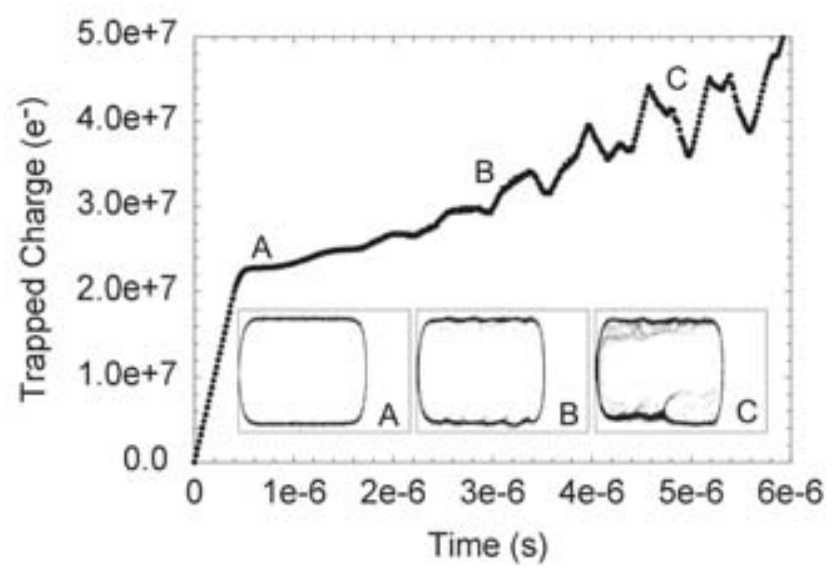

FIGURE 4. The total number of trapped electrons as a function of time during the second stage of injection with inset plots of the $z-v_{z}$ phase space. In this second injection stage, electrons are trapped due to a streaming instability.

waves for short traps.

A conventional theory of two-stream instability, including transverse geometry and temperature, yields the result that the two-stream interactions for beams with parameters appropriate for the center region of the trap is stable. Simulations indicate that a possible explanation for developing the instability is that the perturbations in the vicinity of the cathode are unstable. In this region the velocity is rapidly decreasing and the local plasma density is much higher than in the trap, resulting in an enhanced two-stream interaction. Another remarkable simulation result, under further study, is a strong dependence of dynamics on the energy of electrons as they are emitted from the cathode.

\section{Bubble Stage}

After about ten bounce times, the phase space distribution develops a strongly nonlinear perturbation, which has the visual appearance of a 'bubble'. That is, electrons move around the outside of the disturbance, both gaining and losing energy. as shown in Fig. 5. The bubble's velocity is close to the mean electron velocity in the trap. The bubble persists over many round-trips in the trap, reflecting at the cathode and at the far wall. Numerically, we note that more electrons approaching the cathode along with the bubble have gained energy than have lost energy, leading to momentary depletion of the total charge in the trap. Experimentally, this effect is observable by monitoring the image charge on the inject gate cylinder (Fig 6). Each time the bubble reaches the cathode, the image charge in the inject gate dips slightly. Thus, on top of the increase in the trapped charge due to time-dependent potentials, the rotating bubble induces an oscillation in the trapped charge at, roughly, the bounce frequency. 


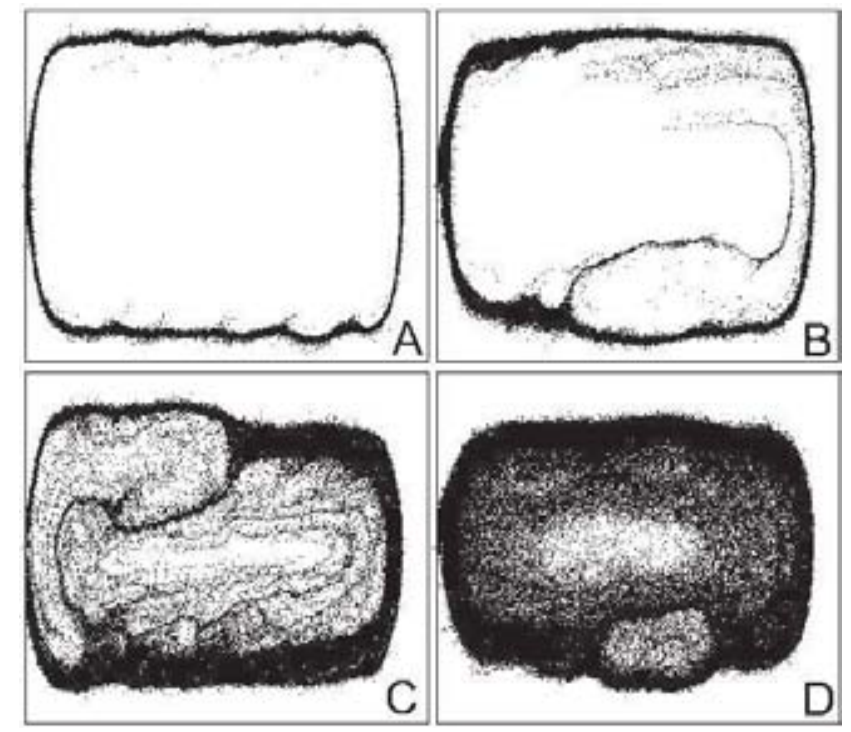

FIGURE 5. PIC code simulation of the $z-v_{z}$ phase space at $3 \mu \mathrm{s}, 6 \mu \mathrm{s}, 13 \mu \mathrm{s}$, and $26 \mu \mathrm{s}$ depicting the development of the bubble instability. Horizontal axis is axial direction with cathode on the left wall and reflecting potential on the right wall (total length of the trap is $36 \mathrm{~cm}$. Axial velocity $v_{z}$ is on vertical axe, extending from $-1.5 \cdot 10^{6} \mathrm{~m} / \mathrm{s}$ to $1.5 \cdot 10^{6} \mathrm{~m} / \mathrm{s}$. The bubble decreases in size over the next $200 \mu \mathrm{s}$, but continues to cause significant perturbations to phase space even beyond this time period. A movie of the phase-space evolution is available at

http://bc1.lbl.gov/CBP_pages/wurtele/UncompressedBubbleMovie1.avi.

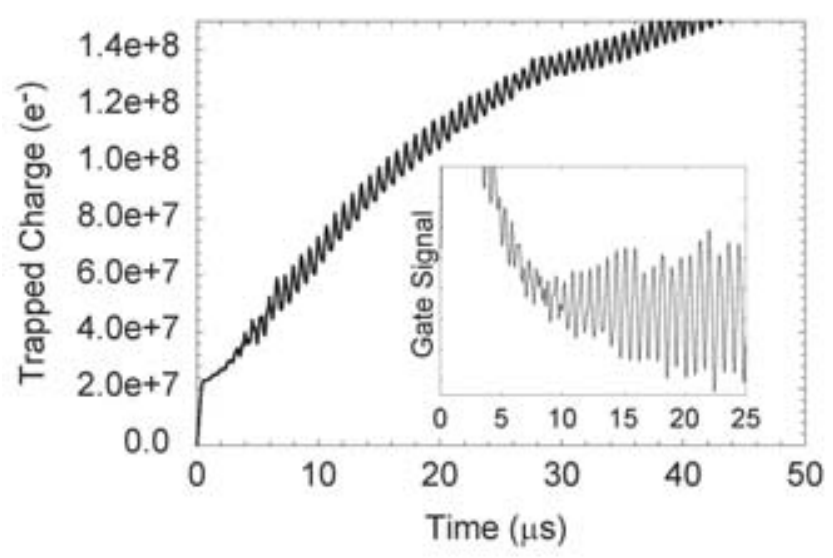

FIGURE 6. Plots of the simulation and experimental data (inset) showing the oscillation in charge associated with the bubble instability. The oscillation in the total charge in the trapping region is manifested by a high frequency signal measured on the inject gate.

Experimental observations and simulation show great similarity in the observable properties of the bubble. In both cases, the bubble begins to form around $10 \mu \mathrm{s}$ after the start of injection. The bubble grows in size, evidenced by the increased amplitude of charge oscillations. The bubble disappears around $70 \mu$ s after the start of injection, and does not reappear. 


\section{Virtual Cathode Formation and Radial Profile Evolution}

Careful study of the dynamics of trapping reveals further complexity, beyond that of large nonlinear perturbations to phase space. As ever more electrons are trapped by energy loss to plasma disturbances, the plasma potential itself becomes a barrier to further trapping. Charges entering the trap are slowed by the space charge potential of the trapped electrons. Simulations show that $\sim 1 \mathrm{~cm}$ in front of the photocathode the potential in the center of the plasma becomes negative. When the potential becomes stongly negative, trapping ceases-there is a self-consistent barrier between the trapped region and the untrapped region. This phenomena is familiar in beam physics as a virtual cathode. In the injection proccess, it appears along with the nonlinear phasespace bubble and oscillates in intensity.

From $10 \mu$ s to $70 \mu$ s, the potential of the virtual cathode becomes increasingly negative. Initially, it fully disappears each cycle, and during the interval that it is not present, new charges enter the system and are trapped. When the virtual cathode reappears it is then more negative. At later times, it no longer vanishes but only weakens during an oscillation. The virtual cathode then limits the injection of additional electrons into the trapping region to those with ever greater initial velocity. Eventually, the system reaches a quasi-equilibrium with few electrons entering or exiting the trapping region.

Beyond $\sim 70 \mu \mathrm{s}$, there is a distinct difference between the evolution of the trapped charge for the equipotential cathode and the filament cathode. The filament cathode potential falls radially as $r^{2}$, due to the spiral design. The same is true of a plasma of constant density. Because of this, the potential profile of the cathode matches the potential profile of the trapped charge at all points. As the virtual cathode grows, it reaches its equilibrium level uniformly over the radius of the electron column.

No match exists for the equipotential photocathode. Here, the virtual cathode begins to form at different times for different radii. The center of the plasma column is more negative than the edge and, thus, the virtual cathode first limits the entrance of new electrons at $r=0$. Charges continue to enter at larger radii, and the virtual cathode then develops outward across the plasma column. The column develops a hollow profile in an attempt to match the constant cathode potential, as shown in figure 7. Initially, the column is of nearly constant density, owing to the uniformly illumination of the cathode. Over the first $30 \mu \mathrm{s}$ of injection, the column density increases from $4 \times 10^{6} \mathrm{~cm}^{-3}$ to $1 \times 10^{7} \mathrm{~cm}^{-3}$ while maintaining a uniform density cross-section to within $10 \%$. and the column then undergoes hollowing. There is good agreement seen in Fig. 7 between the simulation (right) and experiment (left).

Further work needs to be performed to model the physics of injection at longer timescales, where, for example, the diocotron instability is seen in the hollow plasma described above. 


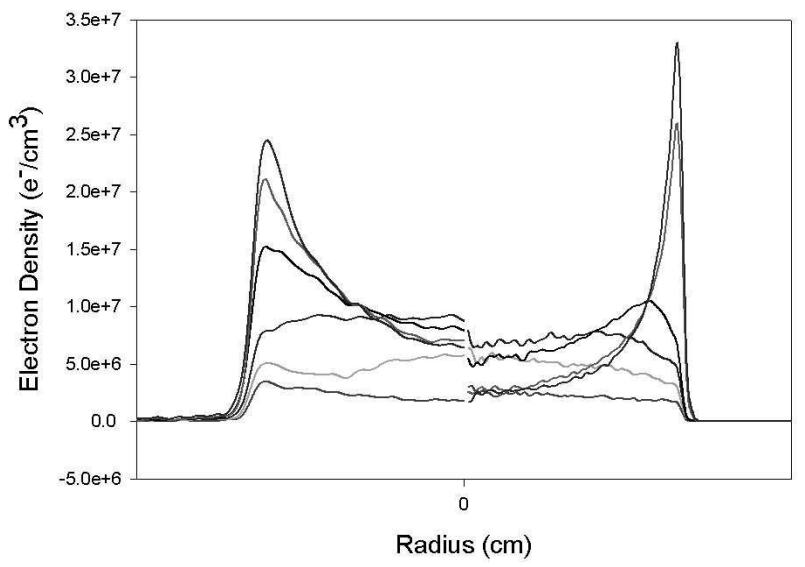

FIGURE 7. Plots of the column cross-section at $1 \mu \mathrm{s}, 10 \mu \mathrm{s}, 30 \mu \mathrm{s}, 50 \mu \mathrm{s}, 500 \mu \mathrm{s}, 1 \mathrm{~ms}, 2 \mathrm{~ms}$ (in order of increasing maximum density) with a $-5 \mathrm{~V}$ photocathode bias. Simulation profiles are shown on the left, experimental profiles on the right. Note that the central density is a maximum at $30 \mu \mathrm{s}$. Beyond $30 \mu \mathrm{s}$ the column begins to hollow due to the mismatched cathode and trapped charge potentials.

\section{ACKNOWLEDGMENTS}

We gratefully acknowledge the assistance of Dr. Peter Mardahl on the XOOPIC code and simulations. This work is supported by the National Science Foundation, the Department of Energy, Division of High-Energy Physics, and the Office of Naval Research.

\section{REFERENCES}

1. See the series of NNP Workshop Proceedings published by AIP.

2. Fine, K. S., Driscoll, C. F., Malmberg, J. H., and Mitchell, T. B., Phys. Rev. Lett., 67, 588 (1991).

3. Hyatt, A. W., Driscoll, C. F., and Malmberg, J. H., Phys. Rev. Lett., 59, 2975 (1987).

4. Durkin, D., and Fajans, J., Rev. Sci. Instrum., 70, 4539 (1999).

5. Driscoll, C. F., and Malmberg, J. H., Phys. Fluids, 19, 760 (1976).

6. Verboncoeur, J. P., Langdon, A. B., and Gladd, N. T., Comp. Phys. Comm., 87, 199 (1995). 